\title{
The attentional cost of external rhythmical cues and their impact on gait in Parkinson's disease: effect of cue modality and task complexity
}

\author{
L. Rochester ${ }^{1,4}$, A. Nieuwboer ${ }^{2}$, K. Baker ${ }^{1}$, V. Hetherington ${ }^{1}$, A.-M. Willems ${ }^{2}$, F. Chavret ${ }^{2}$, \\ G. Kwakkel ${ }^{3}$, E. Van Wegen ${ }^{3}$, I. Lim ${ }^{2}$, D. Jones ${ }^{1}$ \\ ${ }^{1}$ Northumbria University, Newcastle, UK \\ ${ }^{2}$ Katholieke Universiteit, Leuven, Belgium \\ ${ }^{3}$ Vrije Universiteit Medisch Centrum, Amsterdam, The Netherlands \\ ${ }^{4}$ Glasgow Caledonian University, Glasgow, UK
}

Received 10 November 2006; Accepted 5 May 2007; Published online 28 June 2007

(C) Springer-Verlag 2007

\begin{abstract}
Summary Changes in gait performance in 153 subjects with PD using three rhythmical cues (auditory, visual and somatosensory) were measured during a simple walking task and a dual walking task in the home. Subjects were 'on' medication and were cued at preferred step frequency. Accelerometers recorded gait and walking speed, step amplitude and step frequency were determined from raw data. Data were analysed with SAS using linear regression models. Gait performance during a single task reduced with cues in contrast to a dual task where PD subjects appeared to benefit from rhythmical cues (increased speed and step length). Effects were dependent on cue modality with significant improvements for auditory cues compared to others. A significant short-term carry-over effect of cues reduced 3 weeks later. Cues may reduce attentional demands by facilitating attentional allocation, accounting for differences of cue seen during single and dual task. Furthermore cue modality may influence attentional demand which is an important consideration for rehabilitation.
\end{abstract}

Keywords: Cues, gait, Parkinson's disease, attention, dual-task interference, stimulus-response compatibility

\section{Introduction}

Gait deficits are persistent in PD despite optimal pharmacological and drug management and are associated with reduced independence and safety, highlighting the importance of developing alternate approaches to the management of these problems. Evidence from pre-clinical studies, systematic reviews and clinical trials supports the use of

Financial support: European Commission Framework V funding; QLRT2001-00120

Correspondence: Prof. Lynn Rochester, PhD Grad Dip Phys. HealthQWest, Glasgow Caledonian University, Cowcaddens Road, Glasgow, Scotland G4 0BA, UK

e-mail: Lynn.Rochester@gcal.ac.uk cues to improve gait performance in PD (Morris et al. 1996; Thaut et al. 1996; McIntosh et al. 1997; Deane et al. 2001a, b; Howe et al. 2003; Lim et al. 2005; Nieuwboer et al. 2007). External cues have been defined as external temporal or spatial stimuli which facilitate the initiation and continuation of repetitive sequential movements such as gait (Nieuwboer et al. 2007). Different types of cue modality provide information about step frequency or step amplitude and the information from the cue modifies motor responses associated with gait parameters. External information in the form of cues is argued to preferentially activate cortical, parieto-premotor pathways (Cunnington et al. 1995; Debaere et al. 2003). Activation of these pathways may provide a means to avoid the basal ganglia and serve as a compensatory mechanism allowing temporary access to motor programs governing movements such as gait which are problematic in PD.

Gait deficits are exacerbated during the performance of dual tasks in PD because the need to concentrate on both walking and concurrent tasks exceeds available attentional resources (Bond and Morris 2000; Bloem et al. 2001; O'Shea et al. 2002; Rochester et al. 2004). Deficits in executive function documented in PD could add to those difficulties (Litvan 1999; Cools et al. 2001). Executive function is defined as the ability to plan, manipulate information, initiate and terminate activities, and recognise errors (Goverover 2004). Attention is an important part of executive function. Appropriate allocation of attention 
according to changes in task and environmental requirements may be impaired in PD accounting for the gait interference observed during dual and multi-task activities. In support of this, studies have shown significant associations of executive function with dual task performance in PD (Rochester et al. 2004; Yogev et al. 2005). Some evidence from our group has shown that external rhythmical cues can reduce gait interference associated with dual task performance and this may be due to improved allocation of attentional resources (Rochester et al. 2005). Detailed investigation of different cue modalities however has not been undertaken and the optimisation of cueing methods requires further work to increase understanding of the attentional demands and mechanisms of cueing.

We examined three different modalities of external rhythmical cue and tested the cues under single and dual task conditions. As subjects were tested in the home, environmental demands also contributed to the performance with each cue type. Firstly, we hypothesised that the external cues would improve gait performance during single and dual tasks; secondly that the three modalities of external rhythmical cue would result in differences in gait performance as a result of different attentional demands; and thirdly that cues would have immediate carry-over effects which would disappear three weeks later.

\section{Materials and methods}

\section{Subjects}

153 patients with idiopathic PD were recruited from three centres around Northumbria University, Newcastle upon Tyne (UNN), Katholieke Universiteit of Leuven (UNL) and the Department of Neurology at Vrije Universiteit Medical Center of Amsterdam (UNA). The study was approved by the ethics committee of each participating centre. All patients gave informed written consent to the study. Eligibility criteria were: a diagnosis of idiopathic PD, defined following the UK Brain Bank Criteria (Hughes et al. 1992); stable medication usage; Hoehn \& Yahr stage II, III or IV (Hoehn and Yahr 1967); at least one score $\geq 2$ for one or both limbs for either the tremor, rigidity or the bradykinesia items of the UPDRS; able to walk independently; age 18-80 years; no severe cognitive impairments (Mini Mental State Examination (MMSE)) $\geq 24$ (Folstein and Folstein 1975); no other severe neurological, cardiopulmonary or orthopaedic disorders; absence of drug related fluctuations making testing difficult, and no participation in a physiotherapy program two months prior to commencing the trial. Patients were excluded if they had undergone functional neurosurgery. See Table 1 for subject details. This study was conducted as part of a larger effect study of cueing therapy on gait and gait-related mobility reported elsewhere (Nieuwboer et al. 2007).

\section{Experimental protocol}

The effect of three different cue modalities on gait performance was investigated using a test of functional gait which allowed inferences to be made regarding the relative attentional cost of task difficulty and cue modality. Subjects started from standing and were instructed to: walk to a chair placed $6 \mathrm{~m}$ away, pick up a tray with two cups on it (filled to a standard level), turn
Table 1. Characteristics of patient group $(n=153)$

\begin{tabular}{ll}
\hline & Mean (SD) \\
\hline Demography & \\
Male/female* & $88 / 65$ \\
Age & $67.06(7.54)$ \\
PD characteristics & \\
Disease duration & $8.25(5.09)$ \\
Hoehn \&Yahr stages during on II/III/IV* & $71 / 64 / 18$ \\
Freezing of Gait Questionnaire (0-24) & $8.73(5.29)$ \\
Clinical data & \\
Unified Parkinsons Disease Rating Scale -total (on) & $56.03(16.01)$ \\
Levodopa (mg) & $457.82(341.14)$ \\
Mini Mental State Exam & $28.17(1.82)$ \\
Brixton score (1-10) & $3.99(2.22)$ \\
Hospital Anxiety and Depression Scale & $7.20(3.50)$ \\
$\quad$ (depression subscale) & \\
Multidimensional Fatigue Inventory & $62.76(17.93)$ \\
\hline
\end{tabular}

* Expressed as number of patients and $p$-value based on Chi-square test.

around through $180^{\circ}$, carry the tray back to the start position and stop. They were asked to walk at their preferred speed and concentrate equally on all elements of the task. Measurements were taken in the 'on' phase of their medication approximately $1 \mathrm{~h}$ after medication, verified by asking subjects to fill out a visual analogue scale rating how well their medication was working. Eight trials of the test were repeated as follows:

Baseline - No cue $\left(\mathrm{B}^{1}\right)$;

2 cue trials (auditory, visual or somatosensory);

2 cue trials (auditory, visual or somatosensory);

2 cue trials (auditory, visual or somatosensory);

Baseline - No cue $\left(\mathrm{B}^{2}\right)$.

The baseline trials were repeated three weeks later in a subset of subjects $(N=77)$ who were randomised as part of a larger clinical trial to see if any short-term effects of cues were retained. The order of presentation of the three paired cue trials was randomised. Cues were delivered using a prototype cueing device worn on a belt around the waist. The device emitted a flash of light generated by a light emitting diode attached to the subjects own glasses or a pair of clear glasses (VIS); an auditory tone delivered via an earphone (AUD); a somatosensory cue through a miniature vibrating cylinder worn under a wrist band (SS). Subjects were asked to synchronize each step with the rhythmical auditory tone, flash of light or vibration. Frequency of cueing was determined during a $10 \mathrm{~m}$ walk test at preferred walking speed. Gait was measured using an activity monitor (described below) applied in the home before testing. Movement was not restricted in any way by either the monitor or the cueing device and subjects were able to move freely and perform all activities as normal.

\section{Equipment}

The Vitaport Activity Monitor ${ }^{\mathrm{R}}$ (VAM) (TEMEC Instruments Inc) is a valid and reliable tool for measuring gait (White et al. 2006). It was used in PD subjects to determine time and step frequency during the functional test. The activity monitor consists of a portable data recorder worn on a belt around the waist. Movement is measured with accelerometers that record gravitational force and accelerations of the moving limbs and trunk. Five accelerometers were attached to the body: one on each leg positioned on the lateral aspect of the thigh midway between the head of the femur and the mid point of the patella, orientated in the sagittal plane, and three accelerometers were placed on the lower third of the sternum, with the sensors on 
a specially designed block positioned so that they were orientated in the sagittal, longitudinal and transverse planes. The skin was cleansed with an alcohol swab and shaved where necessary. The accelerometers were attached to the skin with Hypafix tape (Hypafix ${ }^{\mathrm{R}}$, BSN Medical). Each accelerometer was connected to the portable battery powered VAM (Vitaport) (TEMEC Instruments Inc) by cables placed under the subject's clothes. Data were sampled at a frequency of $32 \mathrm{~Hz}$ and stored on a removable memory card for later analysis. Data were analysed using a specifically designed software program (Vitagraph) (TEMEC Instruments Inc).

\section{Data analysis}

Walking speed, mean step length and step frequency were estimated from the distance walked by each subject in his/her home, the time taken and number of steps determined from raw data collected by the VAM and described in a previous study (Rochester et al. 2004). Walking speed, step amplitude and step frequency were calculated for two conditions: (1) single = walk; (2) dual task = walk and carry tray. Within each condition, the difference between the cued and final non-cued $\left(\mathrm{B}^{2}\right)$ trials were compared to the first non-cued $\mathrm{B}^{1}$ trial for each gait variable (speed, step amplitude and step frequency). Change in performance between conditions was also calculated for each variable and described as the interference effect using the following equation:

$$
\frac{\text { Condition } 1(\text { single })-\text { Condition } 2(\mathrm{dual})}{\text { Condition } 1(\text { single }) \mathrm{B}_{1}} * 100=\text { Interference effect }
$$

Differences between cue modalities were also compared. Data were analysed separately by a tester who was not involved in data collection and the tester was blinded to cue order for the trials. Data were analysed using linear regression models for repeated measures in Proc Mixed with SAS (version 8.2). Alpha level was tested two-sided and set to $P=0.05$.

\section{Results}

Demographic subject data are shown in Table 1. Subjects had moderate levels of PD (H\&Y II-IV) and were between the ages of 40-80 years. They did not suffer from dementia (MMSE $28.17 \pm 1.82$ ), they did, however, show evidence of poor levels of executive function (Brixton score: $3.99 \pm 2.22$ ) according to scaled scores on the Brixton test. Subject numbers varied during trials due to recording failure or inability to complete testing but on average data from 130 subjects are included in the analysis.

\section{Differences between single and dual task (interference)}

In the first non-cued baseline trial $\left(\mathrm{B}^{1}\right)$ a dual task significantly reduced walking speed by $12.6 \%$ (1.18) $(P \leq$ $0.0001)$ and step length by $12.7 \%(1.16),(P \leq 0.0001)$ but not step frequency (Table 2). All cue modalities significantly reduced the interference effect of the secondary task on gait speed, and step length (Table 2, Interference effect) suggesting that all cues reduced attentional demands.

\section{The effect of cues on gait performance during single task}

The estimated means $( \pm \mathrm{SE})$ for each variable during walking are shown in Table 2 and change scores are in
Table 2. Descriptive data for walk and dual task performance and the interference effect between trials during non-cued and cued trials in Parkinson's disease subjects. Velocity, step amplitude and cadence are expressed as estimated means $\pm S E$

\begin{tabular}{lccc}
\hline & $\begin{array}{l}\text { Single task } \\
(\text { mean SEM) }\end{array}$ & $\begin{array}{l}\text { Dual task } \\
(\text { mean SEM) }\end{array}$ & $\begin{array}{l}\text { Interference effect } \\
\left(\mathrm{DT}-\mathrm{W} / \mathrm{Wb}_{1}\right) * 100\end{array}$ \\
\hline Velocity (m/s) & & & \\
Baseline 1 & $0.96(0.02)$ & $0.84(0.02)$ & $-12.6(1.18)$ \\
Visual & $0.89(0.02)^{*}$ & $0.81(0.02)^{*}$ & $-8.74(1.02)^{*}$ \\
Auditory & $0.94(0.02)$ & $0.87(0.02)^{*}$ & $-6.95(0.86)^{*}$ \\
Somatosensory & $0.92(0.02)^{*}$ & $0.86(0.02)$ & $-5.95(1.03)^{*}$ \\
Baseline 2 & $1.02(0.03)^{*}$ & $0.94(0.03)^{*}$ & $-6.26(1.24)^{*}$ \\
Step Amplitude $(\mathrm{m})$ & & & \\
Baseline 1 & $0.55(0.01)$ & $0.48(0.01)$ & $-12.7(1.16)$ \\
Visual & $0.53(0.01)$ & $0.50(0.01)^{*}$ & $-7.06(0.87)^{*}$ \\
Auditory & $0.55(0.01)$ & $0.51(0.01)^{*}$ & $-7.21(0.74)^{*}$ \\
Somatosensory & $0.55(0.01)$ & $0.51(0.01)^{*}$ & $-6.99(0.85)^{*}$ \\
Baseline 2 & $0.58(0.01)^{*}$ & $0.53(0.01)^{*}$ & $-7.86(1.12)^{*}$ \\
\multicolumn{1}{l}{ Cadence (steps/min) } & & \\
Baseline 1 & $104.85(0.95)$ & $105.20(1.15)$ & $0.38(0.73)$ \\
Visual & $99.62(1.05)^{*}$ & $100.52(1.10)^{*}$ & $1.02(0.51)$ \\
Auditory & $102.08(1.05)^{*}$ & $102.1(1.05)^{*}$ & $0.17(0.49)$ \\
Somatosensory & $100.73(1.07)^{*}$ & $101.77(1.08)^{*}$ & $1.26(0.56)$ \\
Baseline 2 & $105.12(1.00)$ & $105.89(1.08)$ & $1.09(0.55)$ \\
\hline
\end{tabular}

* Denotes a significant different when compared to non-cued baseline trial $\left(\mathrm{B}^{1}\right)$ for single, dual or interference effects. $N=130$ single task; $N=132$ dual task.

Fig. 1. Walking speed decreased with all cues during the single task, and was significant for the visual and somatosensory cue modalities (VIS: $-0.07(0.02) \mathrm{m} / \mathrm{s}$, $P<0.0001$; AUD: $-0.01(0.01) \mathrm{m} / \mathrm{s}, P=0.21$; SS: -0.04 $(0.01) \mathrm{m} / \mathrm{s}, P=0.006)$. The change in speed was related to a small but significant decrease in step amplitude for the visual cue $(-0.01(0.01) \mathrm{m}, P<0.0001)$ compared to the auditory and somatosensory cues (AUD: $0.01(0.006) \mathrm{m}$, $P=0.27$; SS: $-0.001(0.006) \mathrm{m}, P=0.87)$ and significant reductions in step frequency for all cue modalities (VIS: -5.23 (0.76) steps/min, $P<0.0001$; AUD: -2.75 (0.74) steps $/ \mathrm{min}, P=0.0003$; SS: -4.09 (0.76) steps $/ \mathrm{min}, P<$ $0.0001)$. The final non-cued baseline trial $\left(\mathrm{B}^{2}\right)$ showed a short-term carry-over effect of cues with significantly increased walking speed $(06(0.01) \mathrm{m} / \mathrm{s}, P<0.0001)$ and step length $(04(0.006) \mathrm{m}, P<0.0001)$ but no change in step frequency $(0.29(0.66)$ steps $/ \mathrm{min}, P=0.66)$. A comparison of performance between cue modalities indicated that performance with the auditory cue was greater than with the other two modalities for walking speed $(\mathrm{m} / \mathrm{s})$ (AUD v VIS, $P<0.0001$; AUD v SS, $P=0.04$; VIS v $\mathrm{SS}, P=0.01)$ and the visual cue for step amplitude $(\mathrm{m})$ (AUD v VIS, $P<0.0001$; AUD v SS, $P=0.11$; VIS v SS, $P=0.02)$. 

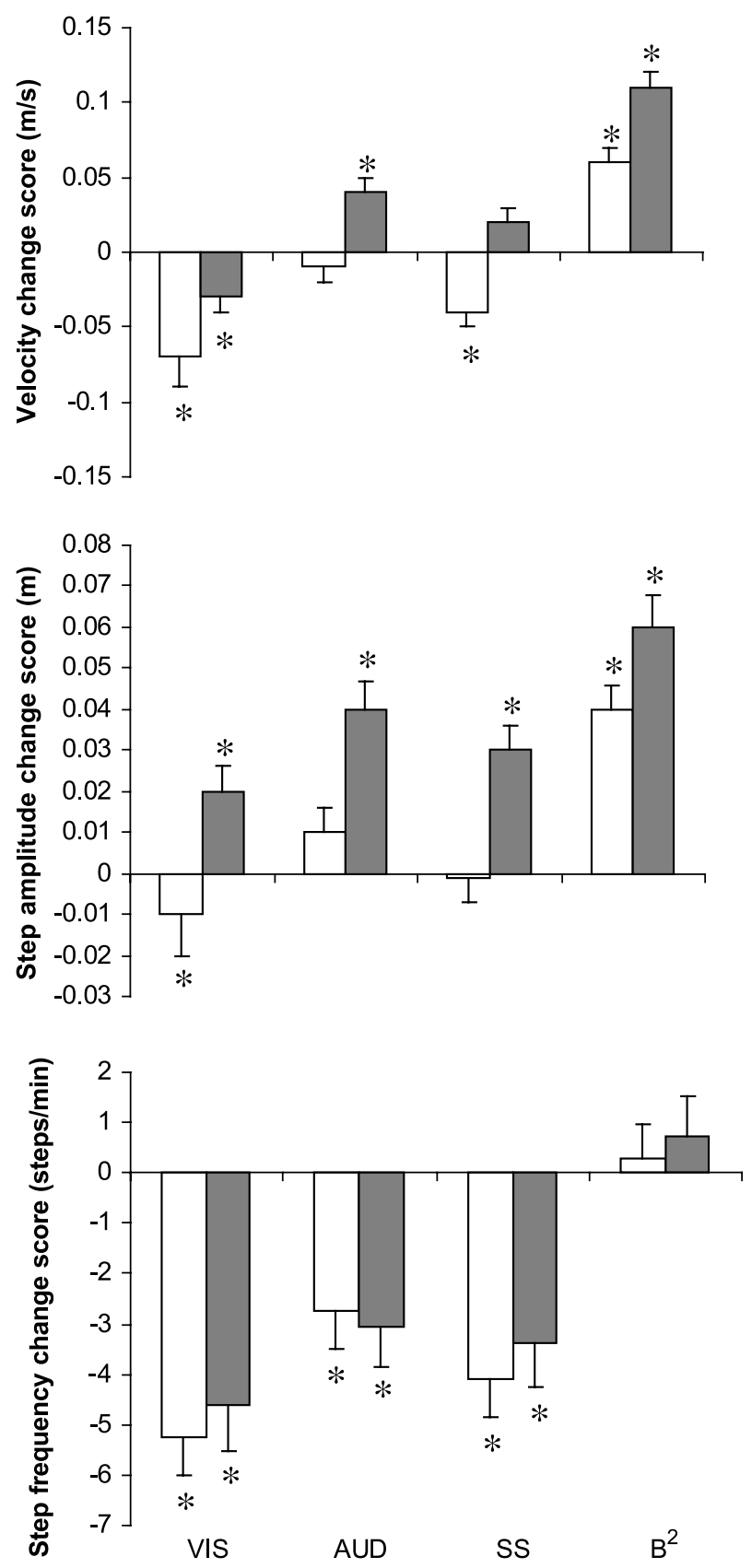

Fig. 1. Change scores (parameter estimates \pm SE) for each cue type from baseline 1 during single (white bars) and dual (grey bars) task. $N=130 .{ }^{*}$ denotes a significant difference from baseline within each task. VIS visual cue, $A U D$ auditory cue, $S S$ somatosensory cue and $B^{2}$ baseline 2

\section{The effect of cues on gait performance during a dual task}

The estimated means $( \pm \mathrm{SE})$ for each variable during the dual task are shown in Table 2 and change scores are in Fig. 1. Walking speed increased with auditory and somatosensory cues during the dual task which was significant for the auditory cue (AUD: $0.04(0.01) \mathrm{m} / \mathrm{s}, P=0.006$; SS:

$0.02(0.01) \mathrm{m} / \mathrm{s} P=0.14)$. This was achieved through a significant increase in step amplitude for both these cue modalities (AUD: $0.04(0.007) \mathrm{m}, P<0.0001$; SS: 0.03 (0.006) $\mathrm{m}, P<0.0001)$, despite a significant decrease in step frequency (AUD: $-3.04(0.80)$ steps $/ \mathrm{min}, P=0.0002$; SS: $-3.38(0.89)$ steps $/ \mathrm{min}, P=0.0002)$. The visual cue showed different responses with a significant decrease in speed $(-0.03(0.01) \mathrm{m} / \mathrm{s}, P=0.03)$ and step frequency $(-4.63(0.91)$ steps $/ \mathrm{min}, P<0.0001)$ and an increase in step amplitude $(0.02(0.006) \mathrm{m}, P=0.002)$. There was a short-term carry-over effect of cues in the final non-cued baseline trial $\left(\mathrm{B}^{2}\right)$ with significantly increased velocity $(0.11(0.01) \mathrm{m} / \mathrm{s}, P<0.0001)$ and step length $(0.06(0.008)$ $\mathrm{m}, P<0.0001)$ and no change in step frequency. A comparison of performance between cue modalities indicated that performance with the auditory cue was greater than the visual cue for walking speed (AUD v VIS, $P<0.0001$; AUD v SS, $P=0.09$; VIS v SS, $P<0.0001)$ and step length (AUD v VIS, $P=0.0003$; AUD v SS, $P=0.18$; VIS $\vee \mathrm{SS}$, $P=0.01)$.

\section{Carry-over effects of cues at $B^{2}$}

Significant increases in speed and step length in $\mathrm{B}^{2}$ were not retained 3 weeks later. Values were no longer significantly different from the first non-cued baseline trial $\left(\mathrm{B}^{1}\right)$ for single (velocity: 0.03 (0.02), $P=0.07$; step length: $0.01(0.01), P=0.44)$ and dual task (velocity: $0.03(0.02)$, $P=0.10$; step length: $0.01(0.01), P=0.22)$.

\section{Discussion}

In agreement with others, the dual task resulted in significantly decreased walking speed and step length compared to the single task showing evidence for increased difficulty with dual tasks in people with PD (Bond and Morris 2000; O'Shea et al. 2002; Rochester et al. 2004). Increased demands of the dual task compete for attentional allocation with the motor task of walking (no longer controlled automatically by the basal ganglia) and utilise attentional resources (Bond and Morris 2000; Rochester et al. 2004).

We hypothesised that external cues would improve gait performance during single and dual tasks and this was partly supported by our findings. External rhythmical cues improved gait during the performance of a secondary motor task and reduced or had minimal effects on walking during a single task, in agreement with our previous findings in a small study (Rochester et al. 2005). Dual task performance in comparison to a single task therefore appeared to benefit more from the presence of cues. This may be explained by 
the fact that subjects simply found the dual task more interesting and were more engaged with it compared to walking alone, thus levels of arousal and performance were heightened relative to the single task condition.

An alternative explanation, however, may relate to an increased reliance on external information under increased task difficulty, particularly where there are coexisting deficits in executive function. Cues could act as an attentional biasing signal favouring cued performance and facilitating more efficient allocation of attentional resources (Behrmann et al. 2004). Where executive function is reduced (as in the present study) subjects may gain benefit from the action of cue to improve attentional allocation, which is further supported by the significant association of executive function with gait interference observed in PD (Rochester et al. 2004; Yogev et al. 2005). The external rhythmic information provided by the cue may reduce attentional load as it informs the motor system about the temporal sequencing of the task rather than needing to internally plan and prepare.

The decrease in interference between single and dual task suggested that cues reduce attentional cost through the preferential activation of parieto-premotor pathways (Debaere et al. 2003), thereby creating spare capacity for performance of concurrent tasks. In support of this, movements occurring in response to external stimuli are shown to involve reduced volumes of brain activity compared to movements that are internally generated (generated by will) (Weeks et al. 2001). In particular there is evidence for reduced activation of the dorsolateral prefrontal cortex and the anterior cingulate cortex which are considered to have a role in executive function and attention (Weeks et al. 2001). These authors suggest that cognitive processing of internally generated movement is more complex than externally cued movement where requirements for movement preparation are reduced. Differences in interference (dual task-single task) however, need to be interpreted together with differences within each condition (visual cues reduced speed for single and dual task) and cautions against the use of a single measure to understand attentional influences on motor control.

Our results confirm our second hypothesis that there would be differences between the modalities of external rhythmical cue as a result of different attentional demands. There were significant differences in performance between the three cue modalities. Visual and somatosensory cues reduced walking speed and step length during the single task, while the auditory cue had little effect but did not impair performance. Step frequency however was significantly reduced for all cue modalities. As step frequency was constrained to preferred rate it may be that subjects had difficulty matching the frequency of the cue. Performance during walking with the auditory cue agrees with previous work showing only small effects of the cue on step length and walking speed during a single task (Howe et al. 2003; Willems et al. 2006).

Reduced effects with cues may relate to the influence of cueing step rate at preferred frequency. In the present study step frequency was determined during a test of straight line walking, which is a more simple task and step frequency may therefore have been overestimated for walking during the test of functional gait. In addition the added influence of testing in the home may account for the small effect sizes seen. Reduced performance, especially with visual cues suggests that in fact this cue modality interfered with gait during single and dual walking conditions. A comparison of the same stimulus modalities in the upper limb also found that subjects responded more easily to the auditory cue than the visual and somatosensory cues and performance with the auditory cue was faster in terms of reaction times (Weeks et al. 2001). The level of congruence of stimulus and response may be an important factor in explaining the differences with cue modalities and the temporal parameter of gait (step frequency) in our study. A flash of light may be difficult to associate with rhythmical stepping in comparison to an auditory or somatosensory modality which may have a more natural association with the temporal qualities of gait. These results are further supported by cue preference from a larger study where $68 \%$ of people with PD chose the auditory and $32 \%$ the somatosensory cue and no subjects preferred the visual cue (Nieuwboer et al. 2007).

Significant carry-over effects of cues on speed and step length during single and dual tasks were seen in post cueing baseline trials $\left(\mathrm{B}^{2}\right)$ and the effects seen were greater without than with cues. These were not accompanied by an increase in step frequency which returned to pre-cueing levels. These responses are difficult to explain but have been reported by others (Kritikos et al. 1995). Effects on speed and step length may result from increased attention (Morris et al. 1996; Cunnington et al. 1999) and indicate that cueing at preferred cadence may have restrained effect sizes of cues. The carry-over effects had gone three weeks later when gait was retested indicating the effects of cueing were short lasting in support of our third hypothesis and in agreement with others (Morris et al. 1996).

The findings of this study are restricted to the external rhythmical cue modalities investigated and also limited to cueing at preferred step frequency. Cues were also only tested when subjects were 'on' medication and results cannot be generalised to when 'off' medication. The effect 
sizes are modest and this may again be related to the frequency of cueing or the fact that this study evaluated the short-term effects only and no training period was used.

This study provides new evidence for the positive effects of cueing on dual task performance and suggests that performance with cues can be generalised to functional activities and also to the home environment in which testing took place. These results together with those of a clinical trial of cueing therapy demonstrating improved balance and no evidence of increased falls as a result of therapy (Nieuwboer et al. 2007) question the association of dual tasks with falls risk. In addition, the differences observed with cue modalities and during the performance of different task complexities highlights the need to increase our understanding of the mechanisms of cueing in order to optimise the delivery of cues as a rehabilitation strategy.

\section{References}

Behrmann M, Geng J, Shomstein S (2004) Parietal cortex and attention. Curr Opin Neurobiol 14: 212-217

Bloem B, Valkenburg V, Slabbekoorn M, van Dijk J (2001) The multiple tasks test. Strategies in Parkinson's disease. Exp Brain Res 137: 478-486

Bond J, Morris M (2000) Goal-directed secondary motor tasks: their effects on gait in subjects with Parkinson's disease. Arch Phys Med Rehabil 81: $110-116$

Cools R, Barker R, Sahakian B, Robbins T (2001) Mechanisms of cognitive set flexibility in Parkinson's disease. Brain 124: 2503-2512

Cunnington R, Iansek R, Bradshaw J, Phillips JG (1995) Movement-related potentials in Parkinson's disease. Presence and predictability of temporal and spatial cues. Brain 118: 935-950

Cunnington R, Iansek R, Bradshaw JA (1999) Movement-related potentials in Parkinson's disease: external cues and attentional strategies. Mov Disord 14(1): 63-68

Deane K, Jones D, Clarke C, Playford D, Ben-Shlomo Y (2001b) Physiotherapy versus placebo or no intervention in Parkinson's disease. The cochrane database of systematic reviews. Issue 3: Art. No. CD 002817

Deane K, Jones D, Ellis-Hill C, Clarke C, Playford E, Ben-Shlomo Y (2001a) Physiotherapy for patients with Parkinson's disease: a comparison of techniques. The cochrane database of systematic reviews. Issue 1: Art. No. CD002815

Debaere F, Wenderoth N, Sunaert S, Van Hecke P, Swinnen SP (2003) Internal vs external generation of movements: differential neural pathways involved in bimanual coordination performed in the presence or absence of augmented visual feedback. Neuroimage 19(3): 764-776

Folstein M, Folstein S (1975) "Mini-Mental State". A practical method for grading the cognitive state of patients for the clinician. J Psychiatr Res 12: $189-198$

Goverover Y (2004) Categorisation, deductive reasoning, and self-awareness: association with everyday competence in persons with acute brain injury. J Clin Exper Neuropsychol 26(6): 737-749
Hoehn M, Yahr M (1967) Parkinsonism: onset, progression and mortality. Neurology (Cleveland) 5: 427-442

Howe TE, Lovgreen B, Cody F, Ashton V, Oldham J (2003) Auditory cues can modify the gait of persons with early-stage Parkinson's disease: a method for enhancing parkinsonian walking performance? Clin Rehabil 17: $363-367$

Hughes A, Daniel S, Kilford L, Lees A (1992) Accuracy of clinical diagnosis of idiopathic Parkinson's disease: a clinico-pathological study of 100 cases. J Neurol Neurosurg Psychiatry 55: 181-184

Kritikos A, Leahy C, Bradshaw J, Iansek R, Phillips J, Bradshaw J (1995) Contingent and non-contingent auditory cueing in Parkinson's disease. Neuropsychologia 33(10): 1193-1203

Lim I, van Wegen E, de Goede C, duetekom M, Nieuwboer A, Willems A, et al (2005) Effects of external rhythmical cueing on gait in patients with Parkinson's disease: a systematic review. Clin Rehabil 19: 695-713

Litvan I (1999) Extrapyramidal disorders and frontal lobe function. In: Miller BL, Cummings JL (eds), The human frontal lobes: functions and disorders. Guilford Press, London, pp 402-421

McIntosh G, Brown S, Rice R, Thaut M (1997) Rhythmic auditory-motor facilitation of gait patterns in patients with Parkinson's disease. J Neurol Neurosurg Psychiatry 62: 22-26

Morris M, Iansek R, Matyas T, Summers J (1996) Stride length regulation in Parkinson's disease. Normalization strategies and underlying mechanisms. Brain 119: 551-568

Nieuwboer A, Kwakkel G, Rochester L, Jones D, Van Wegen E, et al. (The Rescue Consortium) (2007) Impact of a therapeutic cueing program in the home on gait related mobility in Parkinson's disease. A randomised clinical trial. J Neurol Neurosurg Psychiatry 78: 134-140

O'Shea S, Morris M, Iansek R (2002) Dual task interference during gait in people with Parkinson's disease: effects of motor versus cognitive secondary tasks. Phys Ther 82: 888-897

Rochester L, Hetherington V, Jones D, Nieuwboer A, Willems AM, Kwakkel G, et al (2004) Attending to the task: interference effects of functional tasks on walking in Parkinson's disease and the role of cognition, depression, fatigue and balance. Arch Phys Med Rehabil 85(10): 1578-1585

Rochester L, Hetherington V, Jones D, Nieuwboer A, Willems AM, Kwakkel G, et al (2005) The effect of external rhythmical cues (auditory and visual) on walking during a functional task in the home in people with Parkinson's disease. Arch Phys Med Rehabil 86: $999-1006$

Thaut M, McIntosh G, Rice R, Miller R, Rathbun J, Brault J (1996) Rhythmic auditory stimulation in gait training for Parkinson's disease patients. Mov Disord 11(2): 193-200

Weeks R, Honda M, Catalan M, Hallet M (2001) Comparison of auditory, somatosensory, and visually instructed and internally generated finger movements: a PET study. NeuroImage 14: 219-230

White D, Wagenaar R, Ellis T (2006) Monitoring activity in individuals with Parkinson's disease: a validity study. J Neurol Phys Ther 30(1): $12-21$

Willems A, Nieuwboer A, Chavret F, Desloovere K, Dom R, Kwakkel G, et al (2006) The use of rhythmic auditory cues to influence gait in patients with Parkinson's disease, the differential effect for freezers and non-freezers, an explorative study. Disabil Rehabil 28(11): $721-728$

Yogev G, Giladi N, Peretz C, Springer S, Simon E, Hausdorff J (2005) Dual tasking, gait rhythmicity, and Parkinson's disease: which aspects of gait are attention demanding? Eur J Neurosci 22: 1248-1256 